\title{
Symmetries and the conductance of graphene nanoribbons with long-range disorder
}

\author{
Jürgen Wurm, ${ }^{1}$ Michael Wimmer, ${ }^{2}$ and Klaus Richter ${ }^{1}$ \\ ${ }^{1}$ Institut für Theoretische Physik, Universität Regensburg, D-93040 Regensburg, Germany \\ ${ }^{2}$ Instituut-Lorentz, Universiteit Leiden, P.O. Box 9506, 2300 RA Leiden, The Netherlands
}

(Received 25 November 2011; published 8 June 2012)

\begin{abstract}
We study the conductance of graphene nanoribbons with long-range disorder. Due to the absence of intervalley scattering from the disorder potential, time-reversal symmetry (TRS) can be effectively broken even without a magnetic field, depending on the type of ribbon edge. Even though armchair edges generally mix valleys, we show that metallic armchair nanoribbons possess a hidden pseudovalley structure and an effectively broken TRS. In contrast, semiconducting armchair nanoribbons inevitably mix valleys and restore TRS. As a result, in strong disorder metallic armchair ribbons exhibit a perfectly conducting channel, but semiconducting armchair ribbons exhibit ordinary localization. TRS is also effectively broken in zigzag nanoribbons in the absence of valley mixing. However, we show that intervalley scattering in zigzag ribbons is significantly enhanced and TRS is restored even for smooth disorder if the Fermi energy is smaller than the potential amplitude. The symmetry properties of disordered nanoribbons are also reflected in their conductance in the diffusive regime. In particular, we find suppression of weak localization and an enhancement of conductance fluctuations in metallic armchair and zigzag ribbons without valley mixing. In contrast, semiconducting armchair and zigzag ribbons with valley mixing exhibit weak localization behavior.
\end{abstract}

DOI: 10.1103/PhysRevB.85.245418

PACS number(s): 73.63.Nm, 72.80.Vp, 73.20.Fz, 73.23.-b

\section{INTRODUCTION}

The bulk electronic properties of graphene ${ }^{1}$ are modified significantly in nanoscopic samples, where the influence of the edges becomes decisive. Edge effects are most prominent in narrow graphene nanoribbons (GNRs) with the boundary structure determining whether the electronic spectrum is semiconducting (gapped) or metallic (gapless). ${ }^{2-5}$ The nanoribbons with the highest symmetry with regard to the graphene lattice exhibit zigzag and armchair edges, shown in Fig. 1. While zigzag GNRs are always metallic, armchair GNRs are categorized into metallic or semiconducting depending on their width. ${ }^{3-5}$ GNRs of an orientation in between armchair and zigzag have been shown to effectively behave as zigzag GNRs. ${ }^{4,6}$

The first experiments on lithographically defined GNRs failed to find conclusive evidence for these edge effects, ${ }^{7,8}$ but since then great effort has been spent on improving the edges of GNRs: scanning tunneling microscopy has been used to tailor edges, ${ }^{9}$ GNRs have been chemically derived from solution phase, ${ }^{10}$ they have been obtained by unzipping of carbon nanotubes, ${ }^{11-13}$ cut out of graphene sheets by anisotropic etching using nickel clusters ${ }^{14}$ or by sonochemically methods, ${ }^{15}$ or they were self-assembled inside carbon nanotubes. ${ }^{16}$ (For an extended overview we refer to a recent review. ${ }^{17}$ )

In certain situations, for example when the dynamics of a system is chaotic or diffusive, its quantum transport properties are mainly determined by very few symmetries of the system, in particular the presence or absence of time-reversal symmetry (TRS). ${ }^{18}$ TRS is usually broken by magnetic fields. However, Berry and Mondragon ${ }^{19}$ showed that in a (hypothetical) neutrino billiard, TRS is broken even in the absence of a magnetic field. In fact, in this case TRS is broken by the edge of the billiard itself. The Dirac equation used in Ref. 19 to model neutrinos corresponds exactly to the effective low-energy Hamiltonian of a single valley of graphene, prompting efforts to realize such an effective TRS breaking in graphene, for example by different kinds of disorder ${ }^{20,21}$ or edges. ${ }^{22}$

Zigzag GNRs have also been shown to exhibit this kind of effective TRS breaking when only long-range disorder is present, such that the valleys remain uncoupled and a singlevalley Dirac equation description is applicable. In this case, (effective) TRS is broken by the zigzag edges, placing zigzag GNRs with long-range disorder into the unitary symmetry class (no TRS). ${ }^{23}$ The symmetry class was also shown to influence the conductance of zigzag GNRs dramatically: For long-range disorder zigzag GNRs exhibit a perfectly conducting channel (PCC) (i.e., a minimum of one conductance quantum even in the strongly localized regime) whereas they show ordinary localization for short-range disorder that mixes the valleys and restores TRS. ${ }^{23}$ In contrast, armchair GNRs were generally considered to be in the orthogonal symmetry class corresponding to TRS. ${ }^{24,25}$

In this paper, we investigate carefully the symmetries of graphene nanoribbons and their effect on the conductance in the strongly localized and diffusive regime when only long-range disorder (that does not mix the valleys) is present. In particular, we show that in contrast to common belief the symmetry classification of armchair GNRs depends on whether they are metallic or semiconducting. While semiconducting armchair GNRs are found to be in the orthogonal symmetry class, metallic armchair GNRs exhibit a hidden pseudovalley structure, which leads to effective TRS breaking and places metallic armchair GNRs into the unitary symmetry class. This pseudovalley structure also leads to a perfectly conducting channel in metallic armchair GNRs.

In addition, we show that zigzag GNRs can exhibit an unexpected and strong source of intervalley scattering, even for long-range, smooth potentials. When the magnitude of the disorder potential exceeds the Fermi energy, electron-hole puddles are formed and valley scattering can be mediated by the edge state. In this case, TRS is restored in zigzag GNRs 
(a)

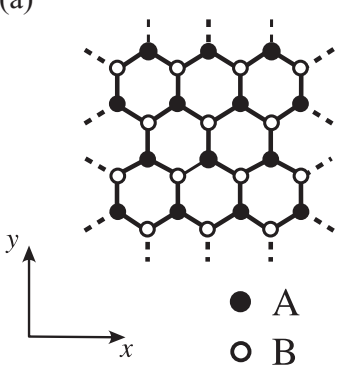

(b)

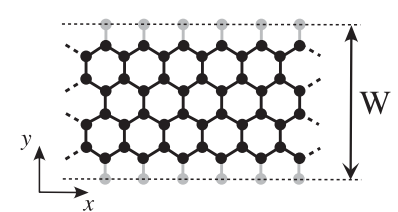

(c)

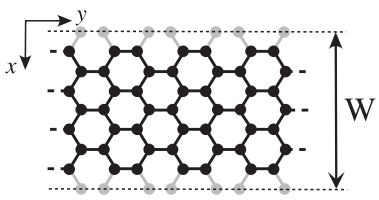

FIG. 1. (a) The graphene honeycomb lattice. The A and B sublattices are indicated as solid and open dots, respectively. (b) zigzag and (c) armchair graphene nanoribbons. The width $W$ of GNRs is measured between the first rows of missing atoms (shown in gray).

and the PCC vanishes. This puts an additional restriction on the disorder potential (apart from being long ranged) in order to realize the unitary symmetry class in zigzag GNRs.

Weak localization effects in the conductance in the diffusive regime of extended bulk graphene have been studied extensively both theoretically and experimentally. ${ }^{20,26-29}$ In contrast, we are aware only of a single theoretical work for (quasi-onedimensional) GNRs that finds weak localization behavior. ${ }^{30}$ Here we study the quantum transport properties of GNRs in the diffusive regime systematically, and show that their behavior is in agreement with their symmetry classifications. In particular, we find suppression of weak localization in zigzag GNRs without intervalley scattering and metallic armchair GNRs. In contrast, zigzag GNRs with strong intervalley scattering and semiconducting armchair GNRs exhibit weak localization behavior. The symmetry classification also reflects itself in the conductance fluctuations of the GNRs.

The paper is organized as follows. In Sec. II we introduce the tight-binding and Dirac Hamiltonian describing the electronic structure of graphene, and briefly describe our numerical method. We investigate the valley scattering properties of a long-range disorder potential in Sec. III, showing that there is an unexpected source of valley scattering for zigzag GNRs. In Sec. IV we classify the different types of GNRs according to their symmetry and study their quantum transport properties. We finally conclude in Sec. V.

\section{GRAPHENE HAMILTONIAN AND QUANTUM TRANSPORT}

\section{A. Hamiltonian}

We describe the electronic structure of graphene using a tight-binding model for the honeycomb lattice [shown in Fig. 1(a)],

$$
H=\sum_{i, j} t_{i, j}|i\rangle\left\langle j\left|+\sum_{i} V\left(x_{i}\right)\right| i\right\rangle\langle i|,
$$

with one orbital $|i\rangle$ per atom and constant hopping $t_{i j}=t$ only between nearest neighbors. We allow for an on-site potential $V(\boldsymbol{x})$ that is evaluated at the positions of the carbon atoms $\boldsymbol{x}_{i}$. A magnetic field is included through the substitution $t_{i j} \rightarrow$

$t \times \exp \left[i \frac{e}{\hbar} \int_{\boldsymbol{x}_{j}}^{\boldsymbol{x}_{i}} d \boldsymbol{x} \boldsymbol{A}(\boldsymbol{x})\right]$, where $\boldsymbol{A}(\boldsymbol{x})$ is the magnetic vector potential.

For a sufficiently smooth potential $V(\boldsymbol{x})$ and in the low-energy limit, excitations with energy $\varepsilon$ obey the Dirac equation

$$
H \Psi=\varepsilon \Psi,
$$

where the Hamiltonian

$$
H=v_{\mathrm{F}} \tau_{0} \otimes(\boldsymbol{\sigma} \cdot \boldsymbol{p})+V(\boldsymbol{x}) \tau_{0} \otimes \sigma_{0}
$$

acts on a four-component spinor wave function

$$
\Psi=\left(\Psi_{\mathrm{A}}, \Psi_{\mathrm{B}},-\Psi_{\mathrm{B}}^{\prime}, \Psi_{\mathrm{A}}^{\prime}\right) .
$$

The Hamiltonian is written in the valley-isotropic form introduced in Ref. 31: $\tau_{i}$ and $\sigma_{i}$ denote the Pauli matrices in valley and sublattice space, respectively $\left(\tau_{0}\right.$ and $\sigma_{0}$ are the respective unit matrices), and $\Psi_{j}$ and $\Psi_{j}^{\prime}$ with $j \in\{A, B\}$ are the wave function amplitudes on the different sublattices in the $K$ and $K^{\prime}$ valley. The Fermi velocity is denoted as $v_{\mathrm{F}}$ and $\boldsymbol{p}=-i \hbar\left(\partial_{x}, \partial_{y}\right)$ is the two-dimensional momentum operator, with the orientation of $x$ and $y$ axis as indicated in Fig. 1(a). A magnetic field is included through the minimal coupling $\boldsymbol{p} \rightarrow \boldsymbol{p}+e \boldsymbol{A}(\boldsymbol{x})$ with $-e$ the electron charge.

\section{B. Numerical quantum transport in the tight-binding model}

To support our analytical predictions, we perform numerical computations of the quantum transport properties of graphene nanoribbons cut out of the graphene lattice [examples of zigzag and armchair graphene nanoribbons are shown in Figs. 1(b) and $1(\mathrm{c})]$.

A potential term $V(\boldsymbol{x})$ is only introduced in a finite part of the system (the scattering region), the remaining parts (i.e., perfect semi-infinite nanoribbons) then serve as leads (with a Fermi energy identical to the scattering region). We compute the lattice Green's function of the system using an adaptive recursive Green's function technique. ${ }^{32}$ From the Green's function we then obtain the scattering matrix using the Fisher-Lee relation for tight-binding systems. ${ }^{33}$ In particular, we compute the amplitudes $t_{n, m}$ for transmission from mode $m$ to mode $n$ between two leads, and the amplitudes $r_{n, m}$ for reflection from mode $m$ to mode $n$ in the same lead. The electrical conductance $G$ is then obtained using the Landauer-Büttiker formalism, ${ }^{34,35}$

$$
G=G_{0} \sum_{n, m}\left|t_{n, m}\right|^{2},
$$

where $G_{0}=2 \frac{e^{2}}{h}$ is the conductance quantum including the spin degree of freedom.

\section{INTERVALLEY SCATTERING IN DISORDERED GRAPHENE}

\section{A. Impurity potential}

We use a model for an impurity potential that is commonly used in the study of disordered graphene (e.g., Refs. 27,36-39). The potential is assumed to consist of a set of independent 

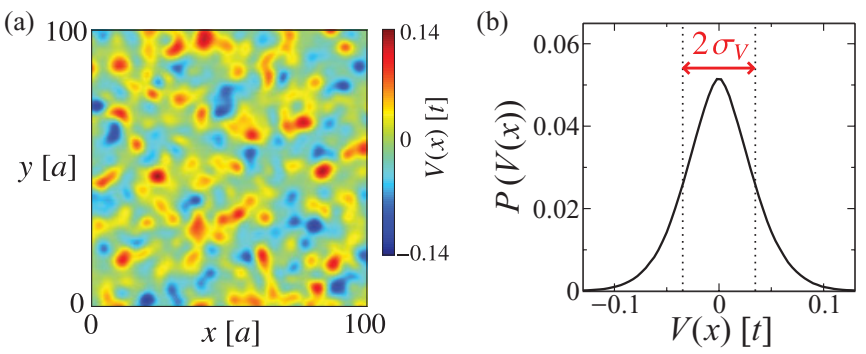

FIG. 2. (Color online) (a) Example of a realization of the impurity potential (6). (b) Numerically computed probability distribution of the value of the potential $V(\boldsymbol{x})$ on a given point $\boldsymbol{x}$. For both (a) and (b), $p_{\text {imp }}=0.05, \xi=2 a$, and $\delta=0.05 t$.

impurities with a Gaussian potential profile

$$
V(\boldsymbol{x})=\sum_{j=1}^{N_{\mathrm{i}}} \delta_{j} \exp \left(-\frac{\left(\boldsymbol{x}-\boldsymbol{X}_{j}\right)^{2}}{2 \xi^{2}}\right) .
$$

Here $\boldsymbol{X}_{j}$ is the position of the $j$ th scattering center. We use $N_{\mathrm{i}}=p_{\text {imp }} N_{\mathrm{a}}$ randomly distributed Gaussian scatterers, where $N_{\text {a }}$ denotes the total number of lattice sites in the disordered region, and $p_{\text {imp }}<1$ is a constant that determines the relative amount of scatterers. Further, we choose the impurity strength $\delta_{j}$ randomly from the interval $[-\delta, \delta]$ and use a constant range $\xi$ for all impurities. Figure 2(a) shows an example of the potential landscape of this type of impurity potential.

The potential (6) can describe both short-range and longrange impurities by varying the impurity range $\xi$. For $\xi \gtrsim a$, the resulting potential varies smoothly on the lattice scale, and the Dirac Hamiltonian (3) is applicable. ${ }^{26}$

The magnitude of the fluctuating impurity potential is best described by its root mean square value. For $\xi \gg a$ (in practice it is enough to have $\xi>a$ ) it is given as

$$
\sigma_{V}=\sqrt{\left\langle V(\boldsymbol{x})^{2}\right\rangle}=\sqrt{\frac{4 \pi p_{\text {imp }}}{3 \sqrt{3}}} \delta \frac{\xi}{a},
$$

where $\langle\ldots\rangle$ denotes an impurity average. Figure 2 shows the probability distribution $P[V(\boldsymbol{x})]$ for finding a particular potential value $V(\boldsymbol{x})$ at a point $\boldsymbol{x}$. The distribution is Gaussianlike, with a width given by $\sigma_{V}$.

In the limit of $\lambda_{\mathrm{F}} \gg \xi \gtrsim a$ (with $\lambda_{\mathrm{F}}$ the Fermi wavelength), the transport mean free path is given in Born approximation as $^{26,36}$

$$
l_{\mathrm{tr}}=\frac{4}{k_{\mathrm{F}} K_{0}}=\frac{2 \sqrt{3} t}{E K_{0}} a
$$

with the dimensionless correlator

$$
K_{0}=\frac{4 \pi}{\left(\hbar v_{\mathrm{F}}\right)^{2}} \sigma_{V}^{2} \xi^{2} .
$$

\section{B. Bulk graphene versus zigzag nanoribbons}

The parameter $\xi$ of the impurity potential (6) determines the smoothness of the potential. It is generally accepted that for $\xi \gtrsim a$ there is only little intervalley scattering. However, the evidence for this was always only indirect, ${ }^{23,27,36,37}$ and no quantitative discussion of intervalley scattering exists for this type of potential. Recently, it has only been attempted to quantify the intervalley scattering for short-range lattice defects. ${ }^{40}$ Since the knowledge of the degree of intervalley scattering will be important in the following section, we first investigate the intervalley scattering for the impurity potential (6). Our findings show that caution must be exercised, as the presence of zigzag edges may lead to enhanced intervalley scattering even if the impurity potential is very smooth.

We can numerically measure the intervalley scattering if we consider a wire along the $x$ direction, using either periodic boundary conditions in $y$ direction (making the system equivalent to an armchair carbon nanotube) or zigzag boundaries. In both cases the valleys $K$ and $K^{\prime}$ project onto different values of the longitudinal momentum $k_{x}$. The scattering states in the leads have a definite Bloch momentum $k_{x}$, and a mode $m$ can thus be uniquely assigned to a valley (this is not possible for armchair ribbons, where the two valleys project on the same momentum). It is then possible to decompose the numerically computed transmission and reflection probabilities into an intravalley and intervalley part. The total probability of reflection into the other valley is given as

$$
R_{\text {inter }}=\sum_{\substack{n \in K, m \in K^{\prime} \\ m \in K, n \in K^{\prime}}}\left|r_{n, m}\right|^{2},
$$

while the total reflection probability is given as $R=$ $\sum_{n, m}\left|r_{n, m}\right|^{2}$. The intervalley transmission probability can be defined analogously.

In Fig. 3 we show the fraction of intervalley scattering in the reflection probability, $R_{\text {inter }} / R$, as a function of the impurity strength $\delta$. The rationale for measuring the intervalley
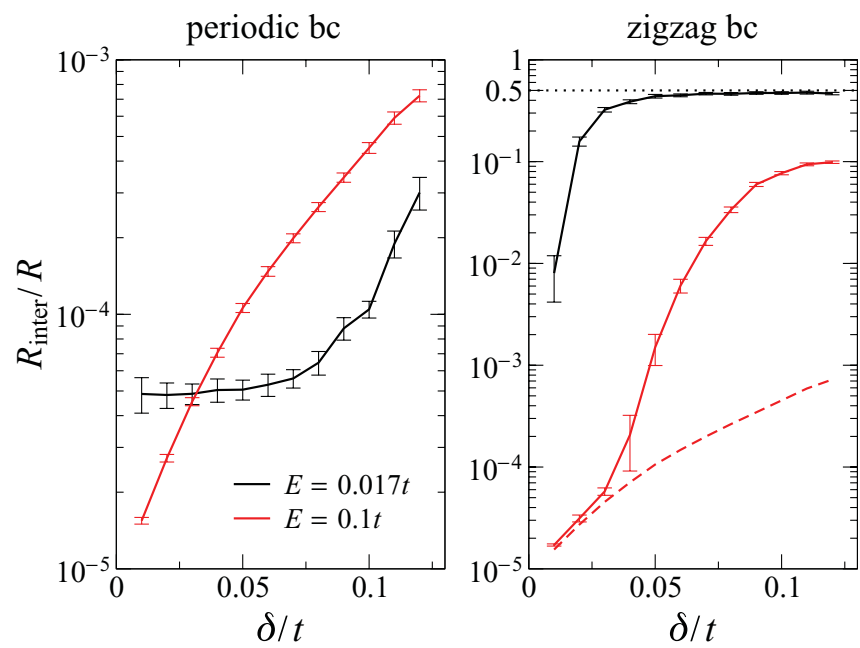

FIG. 3. (Color online) Probability for intervalley scattering in reflection [Eq. (9)] as a function of impurity strength $\delta$ for a ribbon with periodic boundary conditions (left panel) and zigzag boundaries (right panel). The ribbons have width $W \approx 500 a$ and length $L \approx$ $2500 a$, and data is shown for $E=0.017 t$ (black solid lines) and $E=0.1 t$ (red solid lines). For comparison, the right panel (with zigzag boundaries) also contains the data for $E=0.1 t$ with periodic boundary conditions as a red dashed line. The data was obtained by averaging over 100 different impurity configurations with potential parameters $p_{\text {imp }}=0.05$ and $\xi=2 a$. 
scattering in the reflection is that transmission may contain a sizable part of ballistic processes without any scattering; in contrast, reflection only occurs after at least one scattering event. For periodic boundary conditions and $\xi=2 a$ we indeed find only very little intervalley scattering (left panel of Fig. 3). The intervalley scattering rises with increasing impurity strength $\delta$. This is to be expected, as for fixed $\xi$ the potential becomes steeper as $\delta$ is increased, and hence intervalley scattering becomes more likely. Nevertheless, for the given parameters, the fraction of intervalley scattering remained below $10^{-3}$.

However, we obtain a very different picture for zigzag graphene nanoribbons (right panel of Fig. 3). Although we use the same impurity potential as in the case of periodic boundary conditions, we find an intervalley scattering that is three orders of magnitude larger. The only obvious difference is the presence of the zigzag edge. Indeed, it was shown that even a smooth $p$ - $n$ junction in a zigzag nanoribbon strongly scatters valleys, as both valleys are connected by the edge state. ${ }^{41}$ We believe that our numerical findings can be explained fully by the fact that the impurity potential locally leads to many smooth $p$ - $n$ junctions (in the bulk, but also at the zigzag edge), when its magnitude becomes larger than the Fermi energy $E$ : For $E=0.1 t$ and small $\delta$, the intervalley scattering for the zigzag case follows the result obtained with periodic boundary conditions (shown for comparison as a dashed line in the right panel of Fig. 3). For larger $\delta$, when the maxima of the potential become comparable or greater to $E=0.1 t$ (which is already the case around $\delta=0.05 t$ as seen from Fig. 2), the intervalley scattering rate raises rapidly and reaches up to $10 \%$. For smaller Fermi energy $E=0.017 t$, this regime is reached already for much smaller $\delta$, and we find perfect valley mixing close to $50 \%$.

Hence, although the impurity potential itself indeed does not scatter valleys significantly if $\xi \gtrsim a$, caution must be exerted if zigzag edges are present. Intervalley scattering can be very large if the impurity potential locally crosses the Dirac point and hence locally forms $p-n$ junctions. We believe that this unexpected valley scattering may also explain many not understood numerical findings of the past.

We note that the mechanism of the enhancement of intervalley scattering, the formation of local $p$ - $n$ junctions at the zigzag edge, is generic and not due to the particular choice of disorder potential. This is further illustrated in Appendix where we numerically find the same behavior for power-law-correlated disorder.

\section{SYMMETRY AND THE CONDUCTANCE OF GRAPHENE NANORIBBONS}

\section{A. Symmetries and quantum transport in disordered wires}

The quantum transport properties of disordered quantum wires are universal and determined by their symmetries only. ${ }^{18}$ In particular, the symmetry class is determined by the presence or absence of time-reversal symmetry (TRS) $\mathcal{T}$, which is an antiunitary symmetry. A system may belong to one of the three Wigner symmetry classes: unitary if TRS is broken, and orthogonal or symplectic if TRS is present with
$\mathcal{T}^{2}=+1$ or $\mathcal{T}^{2}=-1$, respectively. A system with TRS obeys $\mathcal{T}_{H \mathcal{T}^{-1}}=H$.

Note that a Hamiltonian may possess a TRS that is, however, irrelevant: This is the case if the Hamiltonian decomposes into independent blocks and the symmetry connects only between them. In this case the TRS has no influence on the quantum transport properties (except guaranteeing a degeneracy between the blocks), and the symmetry class is determined by intrablock symmetries only. Below, we identify the appropriate symmetries for the case of graphene nanoribbons.

\section{B. Symmetries of graphene nanoribbons within the Dirac approximation}

\section{Bulk symmetries of graphene and boundary conditions}

The bulk Dirac Hamiltonian (3) commutes with four antiunitary symmetries ${ }^{20,22,26,42}$

$$
\mathcal{T}_{i}=\tau_{i} \otimes \sigma_{y} \mathcal{C} \quad \text { for } \quad i \in 0, x, y, z,
$$

where $\mathcal{C}$ denotes complex conjugation. Each of these antiunitary symmetries can play the role of an (effective) TRS.

It is easy to see that $\mathcal{T}_{y}^{2}=1$, whereas $\mathcal{T}_{i}^{2}=-1$ for $i \in$ $\{0, x, z\} . \mathcal{T}_{y}$ represents the (true) TRS that connects the two valleys. $\mathcal{T}_{x}$ is the valley symmetry that guarantees the Kramer's degeneracy of both valleys ( $\operatorname{since} \mathcal{T}_{x}^{2}=-1$ ). It should be noted that $\mathcal{T}_{0}$ and $\mathcal{T}_{z}$ only differ by a phase in the two valleys and are thus equivalent. In fact, one can write down a whole family of equivalent effective intravalley $\mathrm{TRSs}^{43}$

$$
\mathcal{T}_{0 z}(\vartheta)=\left(\cos \vartheta \tau_{0}+i \sin \vartheta \tau_{z}\right) \otimes \sigma_{y} \mathcal{C}
$$

From this family, a single antiunitary symmetry will survive in the case of metallic armchair nanoribbons, as we will show below. The presence of a magnetic field breaks all four symmetries $\mathcal{T}_{i}$.

The other important symmetries of graphene, chiral symmetry $\left(\tau_{z} \otimes \sigma_{z}\right) H\left(\tau_{z} \otimes \sigma_{z}\right)=-H$, and particle-hole symmetry $\left(\tau_{0} \otimes \sigma_{x}\right) H^{*}\left(\tau_{0} \otimes \sigma_{x}\right)=-H$, are broken by the potential term $V(\boldsymbol{x})$. Hence, we do not expect to see universality classes beyond the three Wigner classes. ${ }^{44}$

It is well known that bulk graphene with long-range scatterers belongs to the symplectic symmetry class. ${ }^{20,26}$ In this case valleys are not mixed and the true TRS $\mathcal{T}_{y}$ as well as the valley symmetry $\mathcal{T}_{x}$ are irrelevant, and the effective intravalley TRS $\mathcal{T}_{0, z}$ determines the symmetry class.

In a graphene nanoribbon, the antiunitary symmetries $\mathcal{T}_{i}$ must also be compatible with the boundary conditions. Boundary conditions in the Dirac equation can be written generally in the form ${ }^{6,45}$

$$
\Psi(\boldsymbol{x})=\mathcal{M}_{b} \Psi(\boldsymbol{x}) \text { for } \boldsymbol{x} \text { on boundary } b,
$$

where $\mathcal{M}_{b}$ is a Hermitian $4 \times 4$ matrix. A graphene nanoribbon is then symmetric with respect to $\mathcal{T}_{i}$, if

$$
\left[\mathcal{M}_{b}, \mathcal{T}_{i}\right]=0
$$

where $[A, B]=A B-B A$ denotes the commutator. Below we now specialize to the cases of zigzag and armchair nanoribbons. 


\section{Zigzag nanoribbons}

The boundary condition for a zigzag GNR reads ${ }^{5,6}$

$$
\mathcal{M}_{b}= \pm \tau_{z} \otimes \sigma_{z} \quad \text { for } b=1,2 .
$$

This boundary condition does not mix valleys, which thus remain a good quantum number. The system is symmetric with respect to $\mathcal{T}_{y}$ and $\mathcal{T}_{x}$, but the boundary condition breaks $\mathcal{T}_{0, z}$. However, since valleys are not mixed for long-range impurities, the valley-offdiagonal symmetries $\mathcal{T}_{x, y}$ are not relevant. Since all intravalley TRSs are broken by the boundary conditions, a zigzag graphene nanoribbon resides in the unitary symmetry class, as shown in Ref. 23.

These considerations hold as long as there is no intervalley scattering due to local $p$ - $n$ junctions at the zigzag edge. If there is, the valleys are strongly mixed, and the only remaining symmetry is the TRS of the tight-binding lattice $\left(\mathcal{T}_{\mathrm{tb}}=\mathcal{C}, \mathcal{T}_{\mathrm{tb}}^{2}=1\right)$. The Dirac equation cannot capture scattering between the valleys via the edge state since a continuous equation cannot represent the finite size of the zigzag GNR Brillouin zone that is at the heart of the scattering mechanism. ${ }^{41}$ The zigzag GNR is then in the orthogonal symmetry class.

\section{Metallic armchair nanoribbons}

The boundary condition for an armchair GNR reads ${ }^{5,6}$

$$
\mathcal{M}_{b}=\boldsymbol{v}_{b} \cdot \boldsymbol{\tau} \otimes \boldsymbol{t} \cdot \boldsymbol{\sigma} ; \quad \boldsymbol{v}_{b}=\left(\sin \vartheta_{b}, \cos \vartheta_{b}, 0\right),
$$

with $b=1,2$, and $t$ points in the direction of the GNR. This boundary condition strongly mixes valleys, and the relative valley angle $\vartheta_{2}-\vartheta_{1}$ between the two boundaries of the armchair GNR depends on the width of the ribbon. For example, for an armchair GNR in $y$ direction as shown in Fig. 1(c), $\vartheta_{b}=-2 K x_{b}$, where $K=4 \pi / 3 a .^{46}$

A metallic armchair nanoribbon has $\vartheta_{1}=\vartheta_{2}=\vartheta$, i.e.,

$$
\mathcal{M}_{1}=\mathcal{M}_{2}=\mathcal{M} \text {. }
$$

In this case, $\boldsymbol{v} \cdot \boldsymbol{\tau}$ commutes with both the Hamiltonian (including disorder) and the boundary condition, and we may choose the solutions of the Dirac equation as eigenstates of $\boldsymbol{v} \cdot \boldsymbol{\tau}$. The solutions can thus be grouped into two new pseudovalleys, $K_{\mathcal{R}}$ and $K_{\mathcal{R}}^{\prime}$, that remain uncoupled for longrange disorder. The pseudovalley description is obtained from the usual valleys by means of the rotation

$$
\mathcal{R}=e^{-i \pi \tau_{x} / 4} e^{-i \vartheta \tau_{z} / 2} .
$$

The metallic armchair boundary condition (16) is symmetric with respect to $\mathcal{T}_{y}$ and $\mathcal{T}_{0 z}(\vartheta)$ with $\vartheta$ equal to the valley angle of the boundary condition, whereas $\mathcal{T}_{x}$ is broken. In the pseudovalley space they take the form

$$
\begin{gathered}
\mathcal{T}_{y}^{\mathcal{R}}=\tau_{y} \otimes \sigma_{y} \mathcal{C}, \\
\mathcal{T}_{0 z}^{\mathcal{R}}(\vartheta)=-i \tau_{x} \otimes \sigma_{y} \mathcal{C},
\end{gathered}
$$

where $\mathcal{T}^{\mathcal{R}}=\mathcal{R} \mathcal{T} \mathcal{R}^{\dagger}$. Hence, both symmetries are completely offdiagonal in valley space and are not relevant for determining the symmetry class in the case of long-range potential. In the absence of any intrapseudovalley TRS, metallic armchair GNRs also belong to the unitary symmetry class. Note that Ref. 47 already discussed effective TRS breaking in the context of the lowest mode of metallic armchair GNRs in a ring
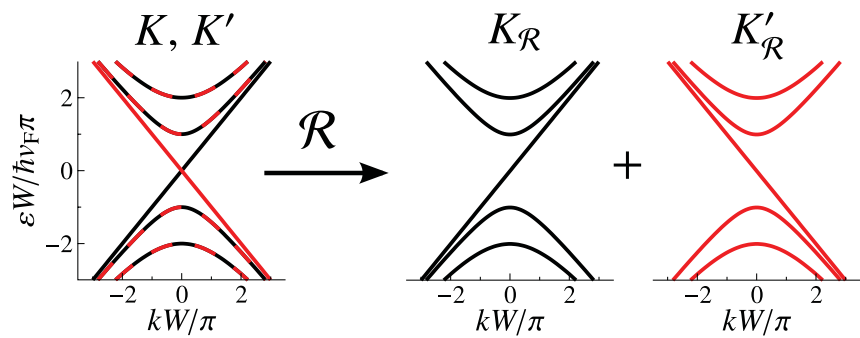

FIG. 4. (Color online) Pseudovalley resolved band structure of a metallic armchair nanoribbon after rotation $\mathcal{R}$ [see Eq. (17)].

geometry. Our analysis shows the more general result that, in the low-energy limit, metallic armchair GNRs belong to the unitary symmetry class without a special geometry and regardless of the number of modes. We will confirm this using numerical simulations below.

A metallic armchair GNR features a band structure $\varepsilon(k)$ (with $k$ the Bloch wave vector) with two nondegenerate gapless linearly dispersing bands and pairs of twofold degenerate hyperbolic bands (shown in Fig. 4). ${ }^{5}$ We can use the conserved antiunitary symmetries to unravel the pseudovalley structure: Both $\mathcal{T}_{y}^{\mathcal{R}}$ and $\mathcal{T}_{0 z}^{\mathcal{R}}(\vartheta)$ lead to ${ }^{48}$

$$
\varepsilon_{K_{\mathcal{R}}}(k)=\varepsilon_{K_{\mathcal{R}}^{\prime}}(-k) .
$$

Hence, the two counterpropagating gapless linear modes are (Kramer's) partners residing in different pseudovalleys. In addition, every pseudovalley contains a set of nondegenerate hyperbolic bands, as shown in Fig. 4.

The pseudovalley structure also reveals that the metallicity (i.e., absence of a gap) of metallic armchair nanoribbons is of topological origin: In pseudovalley space, the boundary condition reads

$$
\mathcal{M}^{\mathcal{R}}=\tau_{z} \otimes \boldsymbol{t} \cdot \boldsymbol{\sigma}
$$

and thus takes the form of infinite mass boundary conditions, ${ }^{6,19}$ with an infinite mass of opposite sign on the opposite edges (if both edges had the same sign of mass, $\mathcal{M}_{1}=-\mathcal{M}_{2}$ ). Hence, the metallic armchair GNR effectively exhibits a domain wall with a sign change in mass and thus supports a gapless linearly dispersing mode. ${ }^{49,50}$

Finally, it must be emphasized that the pseudovalley structure is only valid for energies close to the Dirac point. For higher energies trigonal warping breaks the symmetry between the $K$ and $K^{\prime}$ valley. ${ }^{20,51}$ It introduces terms proportional to $\tau_{z}$ in the Hamiltonian, which then does not commute any more with $\boldsymbol{v} \cdot \boldsymbol{\tau}$ and thus invalidates the notion of pseudovalleys. In fact, the degeneracy of hyperbolic bands (a consequence of the pseudovalley structure) in the tight-binding model is only true close to the Dirac point, as shown in Fig. 5(a). For large energies it is absent [Fig. 5(c)].

\section{Semiconducting armchair nanoribbons}

An armchair GNR is semiconducting, if $\vartheta_{1} \neq \vartheta_{2}$ in Eq. (15) (i.e., if the two boundaries have different boundary conditions)

$$
\mathcal{M}_{1} \neq \mathcal{M}_{2}
$$


(a)

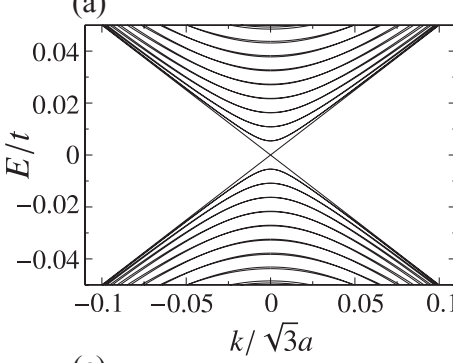

(c)

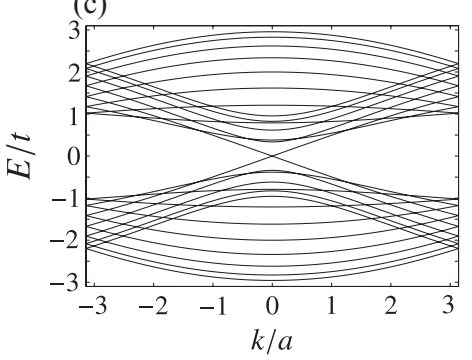

(b)

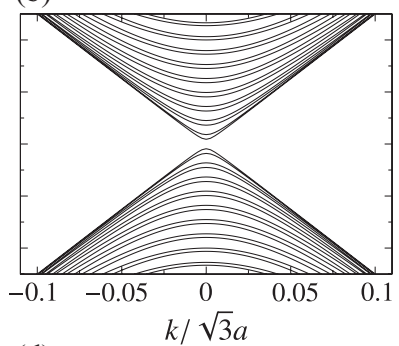

(d)

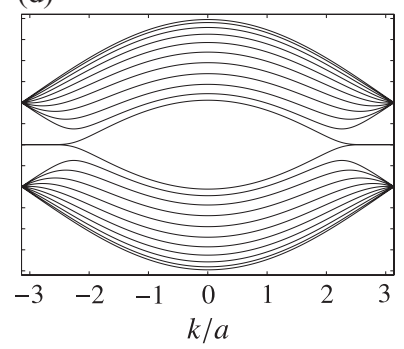

FIG. 5. (a), (b) Tight-binding band structures of wide armchair graphene nanoribbons close to the Dirac point. (a) Metallic armchair GNR with $W=501 a$. The nonlinear bands are approximately degenerate for low energies. (b) Semiconducting armchair GNR with $W=502 a$. (c) Full tight-binding band structure of a narrow metallic armchair GNR with $W=7.5 a$. Clearly the band degeneracy is lifted. (d) Narrow zigzag GNR with $W=19 / \sqrt{3} a$.

In this case, it is not possible to separate any valley structure, and hence there is no degeneracy of bands even close to the Dirac point [Fig. 5(b)]. $\mathcal{T}_{y}$ is the only symmetry of the problem, and hence semiconducting armchair nanoribbons with longrange disorder belong to the orthogonal symmetry class.

Our findings for the different types of GNRs are summarized in Table I.

\section{Perfectly conducting channels \\ 1. Previous work}

One of the most striking features of a zigzag GNR in the absence of intervalley scattering is the presence of a perfectly conducting channel (PCC). ${ }^{23}$ In this case, one of the transmission eigenvalues is exactly one, such that $G / G_{0} \geqslant 1$, regardless of the strength of the disorder. As explained in Ref. 23, within a single valley, a zigzag GNR has unequal numbers $p, q$ of right-moving and left-moving channels, respectively. This can only occur in the absence of TRS and

TABLE I. Summary of symmetry classification of GNRs with long-range disorder and energies close to the Dirac point. For zigzag GNRs we must distinguish whether there is intervalley scattering due to local $p-n$ junctions at the zigzag edge.

\begin{tabular}{ll}
\hline \hline GNR & Symmetry class \\
\hline $\begin{array}{l}\text { Metallic armchair } \\
\text { Zigzag, no intervalley scattering due to } \\
p-n \text { junctions at edge }\end{array}$ & $\begin{array}{l}\text { Unitary } \\
\text { Unitary }\end{array}$ \\
$\begin{array}{l}\text { Zigzag, intervalley scattering due to } \\
p \text { - } n \text { junctions at edge }\end{array}$ & Orthogonal \\
Semiconducting armchair & Orthogonal \\
\hline \hline
\end{tabular}

limits the conductance from below, ${ }^{44}$

$$
G / G_{0} \geqslant|p-q| \text {. }
$$

In a zigzag GNR the difference in right and left movers, $\mid p-$ $q \mid=1$, is due to the zigzag edge state that connects the two valleys [see Fig. 5(d)].

\section{Armchair nanoribbons}

From our previous analysis, we found that metallic armchair GNRs also exhibit broken (effective) TRS and have $|p-q|=$ 1 within a single pseudovalley (Fig. 4). The inequality of left and right movers in this situation is associated with the linearly dispersing modes of opposite velocity in the two pseudovalleys. Hence, we also expect a PCC in this situation. In contrast, a semiconducting armchair GNR belongs to the orthogonal group and we expect to see ordinary localization.

This is confirmed by our numerical simulations in Fig. 6(a): We clearly see different localization behavior for semiconducting and metallic armchair GNRs, with the latter saturating at $G / G_{0}=1$ for long ribbons, and thus exhibiting a PCC. It should be emphasized that this PCC is not identical to a single
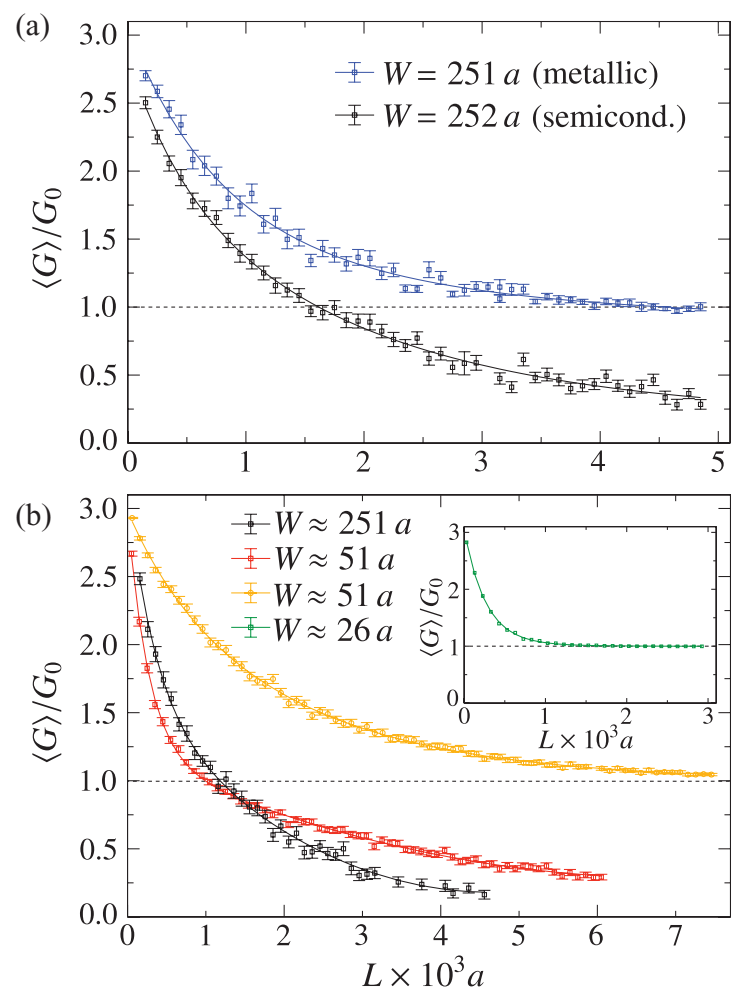

FIG. 6. (Color online) Average conductance of (a) armchair and (b) zigzag GNRs as a function of ribbon length. In all systems the Fermi energy corresponds to three open channels. (a) Semiconducting armchair GNR (black) with $W=251 a$ and metallic armchair GNR (blue) with $W=252 a$. In both cases, Fermi energy $E=0.017 t$ and the disorder parameters are $p_{\text {imp }}=0.05, \xi=2.0 a$ and $\delta=0.08 t$. (b) Zigzag GNR with different widths and disorder parameters (with $p_{\text {imp }}=0.05$ and $\xi=2.0 a$ fixed). Black: $W=434.5 / \sqrt{3} a \approx 251 a$, $E=0.022 t, \delta=0.08 t$. Red: $W=88 / \sqrt{3} a \approx 51 a, E=0.11 t$, $\delta=0.08 t$. Orange: $W=88 / \sqrt{3} a \approx 51 a, E=0.11 t, \delta=0.04 t$. Green (inset): $W=44.5 / \sqrt{3} a \approx 26 a, E=0.22 t, \delta=0.08 t$. The data in (a) and (b) was averaged over 50-200 impurity configurations. 
channel of the GNR (i.e., the edge state in a zigzag GNR or the linearly dispersing mode in a metallic armchair GNR), instead the unit transmission eigenvalue corresponds to a superposition of all channels.

A previous numerical study ${ }^{25}$ of metallic armchair GNRs with long-range disorder found an only approximately unit conductance quantization in the single-mode regime, and the absence of a PCC in the multichannel regime. In contrast, our numerical data shows a PCC even in the multimode regime [three open channels in Fig. 6(a)]. This apparent contradiction is resolved by noting that the simulations of Ref. 25 used very narrow GNRs $(W=7.5 a \approx 1.8 \mathrm{~nm})$, where the second channel only opens at energies far from the Dirac point [around $0.3 t$ in Fig. 5(c)]. For those high energies, trigonal warping breaks the pseudovalley structure and the PCC vanishes.

\section{Zigzag nanoribbons}

In our simulations we have carefully chosen the parameters of the disorder potential such that bulk valley scattering is indeed negligible, as shown in Sec. III B. However, there we also observed that valley scattering could be large in the presence of zigzag edges. This has also consequences for the PCC in zigzag GNRs: In Fig. 6(b) we show the average conductance as a function of ribbon length for various energies, disorder parameters and width. In particular, zigzag GNRs do not show a PCC for a disorder where a metallic armchair GNR very well did [black line in Fig. 6(b) and blue line in Fig. 6(a), respectively]. Only if the amplitude of the disorder potential is smaller than the Fermi energy (i.e., if no local $p$ - $n$ junctions are formed) a PCC can be observed [orange line in Fig. 6(b)]. However, as the disorder has to be chosen weaker, the conductance saturates only for very long GNRs. This breakdown of the PCC due to valley scattering mediated through the zigzag edge state has not been observed in previous studies that dealt with narrow ribbons $(W \approx 5 a \approx 1.2 \mathrm{~nm}$ in Ref. 23) at energies further away from the Dirac point [for an example of a PCC in this case, see inset of Fig. 6(b)].

\section{Zigzag versus metallic armchair nanoribbons}

In summary, zigzag and metallic armchair GNRs both exhibit a PCC. In metallic armchair GNRs, its observation requires small energies close to the Dirac point; it vanishes when the Fermi energy is larger and in a regime where trigonal warping becomes effective. In contrast, for zigzag GNRs the Fermi energy must be larger than the potential amplitude, otherwise the valleys are coupled and the PCC vanishes. Hence, in metallic armchair GNRs the Fermi energy should be smaller than an intrinsic energy scale (trigonal warping), whereas in zigzag GNRs the Fermi energy should be larger than an extrinsic energy scale (disorder potential) in order to observe the effective TRS breaking and a PCC.

\section{Magnetoconductance}

Apart from the PCC that reveals itself mainly in the strongly localized regime, the symmetry class also influences the conductance in the diffusive regime. Due to quantum-coherence corrections, the conductance can be either smaller [weak localization (WL)], larger [weak antilocalization (WAL)], or equal to the classical conductance for the orthogonal, symplectic, and unitary symmetry classes, respectively. ${ }^{18}$ These quantum coherence corrections reveal themselves in the magnetoconductance, in particular in the change of the disorder averaged conductance with magnetic field

$$
\langle\delta G(B)\rangle=\langle G(B)\rangle-\langle G(B=0)\rangle,
$$

where $B$ is a magnetic field perpendicular to the GNR and $G(B)$ the conductance for given field $B$. Since a magnetic field breaks TRS, every GNR is in the unitary symmetry class for large enough $B$. Hence, for large enough $B,\langle\delta G(B)\rangle>$ 0 (WL) in the orthogonal class, $\langle\delta G(B)\rangle<0$ (WAL) in the symplectic class, and $\langle\delta G(B)\rangle=0$ (suppressed WL) in the unitary class.

Figure 7 summarizes our results of magnetotransport simulations for zigzag and armchair GNRs. As expected from our symmetry considerations in Sec. IV B, we observe WL behavior for semiconducting armchair GNRs (black curve), whereas the WL correction is suppressed in the metallic armchair GNR (blue curve). The results for the zigzag GNRs demonstrate again the importance of intervalley scattering: For small Fermi energy (red line) the intervalley scattering is large [parameters as for the black line in Fig. 3(b)] leaving only the tight-binding TRS $\mathcal{T}_{\text {tb }}$. Thus we observe WL behavior just as in the case of the semiconducting armchair GNR, as both belong to the orthogonal symmetry class. Only if valley scattering is suppressed for larger energies (violet curve), we also observe a suppression of the WL correction, as expected from the unitary symmetry class (there is some residual intervalley scattering in this case, preventing complete suppression as in the metallic armchair GNR).

Previous studies ${ }^{20,30}$ assumed that the role of edges is only to introduce valley scattering and hence expected WL behavior in GNRs. In contrast, our study has shown that the type of edge, and even the distance between opposite edges is crucial to

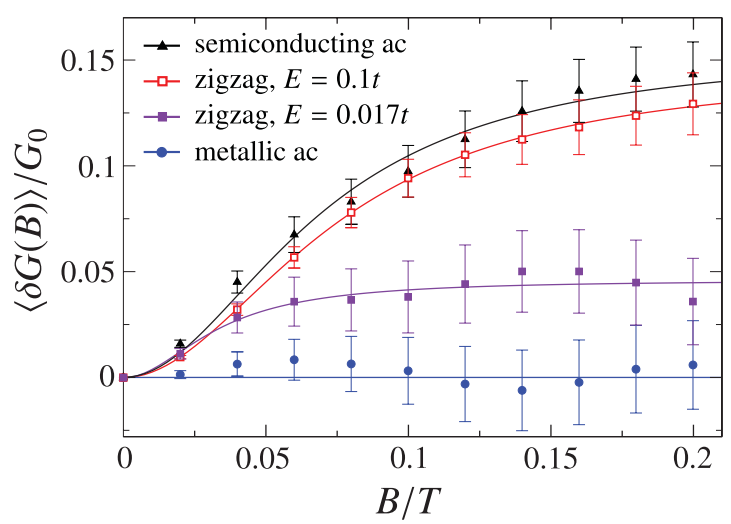

FIG. 7. (Color online) Normalized magnetoconductance of disordered GNRs: semiconducting armchair GNR (black) with $W=$ $502 a$, and $E=0.017 t$ (six open channels), metallic armchair GNR (blue) with $W=501 a$, and $E=0.017 t$ (seven open channels), and zigzag GNR with $W=865.5 / \sqrt{3} a \approx 500 a$ and $E=0.017 t$ (red, five open channels) and $E=0.1 t$ (violet, 35 open channels). In all cases, the GNR length was $L \approx 2500 a$, and the disorder parameters $p_{\text {imp }}=0.05, \xi=2.0 a$ and $\delta=0.08 t$ [as in Fig. 6(a)]. The data was averaged over 600 impurity configurations, lines are guide to the eye. 
understand the magnetoconductance of GNRs in the quantum regime.

Random matrix theory ${ }^{18}$ (RMT) predicts a universal value of the quantum-coherence correction in the limit of a large channel number. This value only depends on how far the system has approached the diffusive limit measured by the parameter $s=2 L / \pi l_{\text {tr }}$. From Eq. (8) we obtain $s \approx 1.6$ for the chosen disorder parameters (see caption of Fig. 7), which agrees well with the value estimated from the average conductance in the simulation $(s \approx 2)$. The value of the WL correction in the orthogonal symmetry class is then ${ }^{18}$ $\lim _{B \rightarrow \infty}\left\langle\delta G(B) / G_{0}\right\rangle \approx 0.26$. The WL correction obtained from our numerical simulations agrees reasonably with this prediction, given that the number of channels is still small.

\section{E. Universal conductance fluctuations}

In addition to the quantum-coherence correction to the (average) conductance, RMT also predicts universal values of the conductance fluctuations $\operatorname{Var}\left(G / G_{0}\right)$ [universal conductance fluctuations (UCF) $].{ }^{18}$ Both zigzag and metallic armchair GNRs are in the unitary symmetry class but have degenerate (pseudo)valleys. As a consequence, we expect UCFs of four times the universal value of the unitary class. In magnetic field, the (pseudo)valleys remain independent, but their degeneracy is broken, since all of the antiunitary symmetries (10) are broken by magnetic field. Hence the UCFs take twice the value of the unitary class. A semiconducting armchair GNR does not allow for a decomposition into independent blocks and thus the UCFs take the value of the orthogonal and unitary class in the absence and presence of a magnetic field, respectively. Since the UCFs in the orthogonal symmetry class are twice as large as in the unitary symmetry class, the UCFs in zigzag and metallic armchair GNRs are twice as large as for semiconducting armchair nanoribbons, both in the absence and presence of a magnetic field.

Figure 8 shows the universal conductance fluctuations as obtained from our numerical simulations. We indeed observe that the UCFs of metallic armchair GNRs and zigzag GNRs

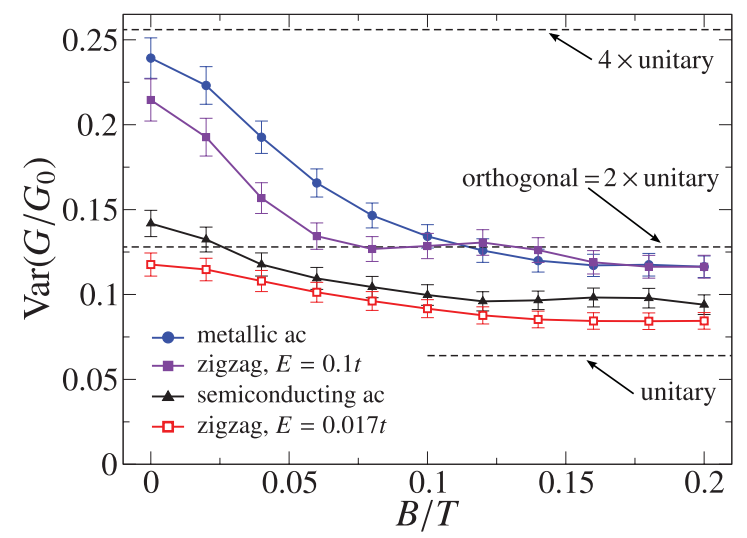

FIG. 8. (Color online) Universal conductance fluctuations as a function of magnetic field for semiconducting armchair (ac) GNRs (black), metallic armchair GNRs (blue), and zigzag GNRs (red: $E=$ $0.017 t$, large intervalley scattering; violet: $E=0.1 t$, small intervalley scattering). The dashed lines show the RMT predictions for the value of the UCFs for $s=1.6$. Parameters as in Fig. 7. with little intervalley scattering are always significantly larger than the UCFs of semiconducting armchair GNRs and zigzag GNRs with large intervalley scattering. We even find good quantitative agreement with the RMT values. Only the UCFs for semiconducting armchair GNRs and zigzag GNRs with large intervalley scattering are somewhat larger than the theoretical prediction, probably due to the still small number of channels.

\section{CONCLUSION}

We have carefully investigated the symmetry classifications of graphene nanoribbons with long-range disorder in the Dirac limit, and studied their imprints on the conductance. Table I summarizes our findings.

In principle, all of the considered graphene nanoribbons are time-reversal symmetric in the absence of a magnetic field. However, if intervalley scattering is absent (hence the condition of long-range disorder), this true TRS that connects the two valleys is irrelevant, and the type of boundary is decisive for the symmetry properties.

In particular, we have found that in the case of armchair GNRs (that up to now were generally assumed to be in the orthogonal symmetry class) it is necessary to distinguish between semiconducting and metallic variants: While semiconducting armchair GNRs inevitably mix valleys and thus belong to the orthogonal symmetry class, metallic armchair GNRs have a hidden pseudovalley structure that together with the boundary conditions places them into the unitary symmetry class.

Zigzag graphene nanoribbons have already previously ${ }^{23}$ been identified to belong to the unitary symmetry class. However, we have shown for this classification it is necessary that the Fermi energy is larger than the disorder potential fluctuations. Otherwise, local $p$ - $n$ junctions at the zigzag edge act as strong intervalley scatterers. ${ }^{41}$ We have demonstrated numerically that the intervalley scattering due to this mechanism can lead to complete valley mixing, although the disorder potential alone would not scatter valleys. Hence, for zigzag nanoribbons to be in the unitary class it is not enough to be in the Dirac limit and to have long-range disorder, there is also a restriction on the magnitude of the potential with respect to the Fermi energy.

The symmetries of the GNRs also have a strong influence on their quantum transport properties. In a metallic armchair GNR, the pseudovalley structure manifests itself most conspicuously in a perfectly conducting channel (i.e., a lower bound of one conductance quantum even in a strongly disordered nanoribbon).

The perfectly conducting channel reveals itself most clearly in the strongly localized regime, but the symmetries of the GNRs also manifest themselves in the diffusive regime. We showed that weak localization is strongly suppressed in metallic armchair GNRs as well as zigzag GNRs with little intervalley scattering. In contrast, semiconducting armchair GNRs and zigzag GNRs with a disorder potential amplitude larger than the Fermi energy exhibit weak localization (instead of weak antilocalization expected for bulk graphene with longrange disorder) due to intervalley scattering at the armchair edges and local $p$ - $n$ junction at the zigzag edge, respectively. 
In addition, the interplay of symmetry classes and the degeneracy of the (pseudo)valley structure of metallic armchair GNRs and zigzag GNRs with little intervalley scattering leads to larger conductance fluctuations than in semiconducting armchair GNRs and zigzag GNRs with intervalley scattering.

\section{ACKNOWLEDGMENTS}

We thank Inanc Adagideli for many helpful conversations. The numerical simulations have been performed on the Höchstleistungsrechner Bayern II (HLRB II) at the LeibnizRechenzentrum, Munich. M.W. was supported by a European Research Council Advanced Investigator Grant and the Eurocores program EuroGraphene. J.W. and K.R. acknowledge funding from the German Research Foundation (DFG) within GRK 1570.

\section{APPENDIX: INTERVALLEY SCATTERING AND ZIGZAG EDGES FOR COULOMB DISORDER}

In Sec. III B we discussed that the presence of zigzag edges can drastically enhance intervalley scattering when local $p-n$ junctions are formed at the edge due to the disorder amplitude being comparable to the Fermi energy. In order to further demonstrate the generality of this mechanism, we show here that this behavior persists also for other types of disorder potentials, in particular power-law-correlated potentials.

For this we use a disorder potential consisting of randomly placed Coulomb potentials

$$
V(\boldsymbol{x})=\sum_{j=1}^{N_{\mathrm{i}}} \frac{\delta}{\sqrt{\left(\boldsymbol{x}-\boldsymbol{X}_{j}\right)^{2}+d^{2}}}-\langle V\rangle .
$$

$\boldsymbol{X}_{j}$ is the position of the $j$ th impurity, and $d$ its distance from the graphene sheet. We use $N_{\mathrm{i}}=p_{\text {imp }} N_{\mathrm{a}}$ randomly distributed scatterers, where $N_{\mathrm{a}}$ denotes the total number of lattice sites in the disordered region, and $p_{\text {imp }}<1 . \delta$ is used to control the strength of the impurities (and for real Coulomb impurities determined by the dielectric constant and the charge of the impurity). In order to avoid trivial shifts of the Fermi energy, we choose the disorder potential such that it has zero mean [obtained by subtracting the mean in Eq. (A1)]. (a)

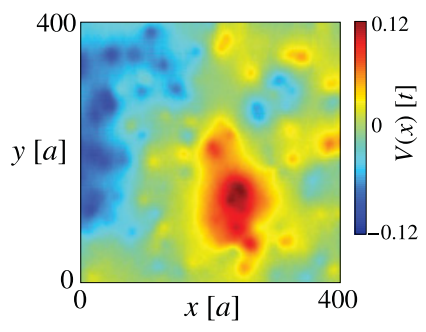

(b)

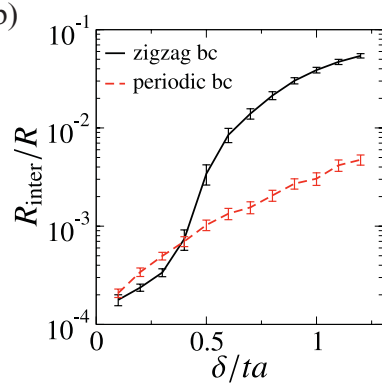

FIG. 9. (Color online) (a) Example of a realization of the Coulomb-like impurity potential (A1) for $\delta=0.5 \mathrm{ta}$. Note the different spatial scale compared to Fig. 2(a). (b) Probability for intervalley scattering in reflection as a function of impurity strength $\delta$ [Coulomb-like impurities (A1)]. The data for zigzag boundaries (black solid line) and for periodic boundary conditions (red dashed line) were obtained by averaging over 100 impurity configurations. The ribbons had width $W \approx 500 a$ and length $L \approx 2500 a$, the Fermi energy was $E=0.1 t$. In both (a) and (b), $d=10 a$ and $p_{\text {imp }}=$ $5 \times 10^{-4}$.

In Fig. 9(a) we show an example realization of the Coulomb-like disorder potential, showing variations on a larger length scale compared to Gaussian disorder potential in Fig. 2(a). Nevertheless, we see very similar behavior for the intervalley scattering [Fig. 9(b)]: As for the Gaussian disorder potential, we observe a similar intervalley scattering probability for both zigzag and periodic boundary conditions at small impurity strengths $\delta$. However, when the potential amplitude becomes comparable to the Fermi energy [around $\delta=0.4-0.5 t$, cf. Fig. 9(a)], the intervalley scattering rate raises dramatically for zigzag boundaries and is an order of magnitude larger than for periodic boundary conditions.

The reason is again the formation of local $p$ - $n$ junctions at the zigzag edge, which happens generically for every type of disorder, when the disorder potential amplitude becomes comparable to the Fermi energy. The relative enhancement due to the zigzag edges depends on the geometry of the ribbon and the details of the potential: For example, it is smaller in Fig. 9(b) compared to Fig. 3, as there are fewer local $p-n$ junctions due to the slower fluctuations of the disorder potential.

${ }^{1}$ A. H. Castro Neto, F. Guinea, N. M. R. Peres, K. S. Novoselov, and A. K. Geim, Rev. Mod. Phys. 81, 109 (2009).

${ }^{2}$ K. Tanaka, S. Yamashita, H. Yamabe, and T. Yamabe, Synth. Met. 17, 143 (1987).

${ }^{3}$ M. Fujita, K. Wakabayashi, K. Nakada, and K. Kusakabe, J. Phys. Soc. Jpn. 65, 1920 (1996).

${ }^{4}$ K. Nakada, M. Fujita, G. Dresselhaus, and M. S. Dresselhaus, Phys. Rev. B 54, 17954 (1996).

${ }^{5}$ L. Brey and H. A. Fertig, Phys. Rev. B 73, 235411 (2006).

${ }^{6}$ A. R. Akhmerov and C. W. J. Beenakker, Phys. Rev. B 77, 085423 (2008).

${ }^{7}$ M. Y. Han, B. Özyilmaz, Y. Zhang, and P. Kim, Phys. Rev. Lett. 98, 206805 (2007).
${ }^{8}$ Z. Chen, Y.-M. Lin, M. J. Rooks, and P. Avouris, Physica E 40, 228 (2007).

${ }^{9}$ L. Tapaszto, G. Dobrik, P. Lambin, and L. Biro, Nature Nanotech. 3, 397 (2008).

${ }^{10}$ X. Li, X. Wang, L. Zhang, S. Lee, and H. Dai, Science 319, 1229 (2008).

${ }^{11}$ D. V. Kosynkin, A. L. Higginbotham, A. Sinitskii, J. R. Lomeda, A. Dimiev, B. K. Price, and J. M. Tour, Nature (London) 458, 872 (2009).

${ }^{12}$ L. Jiao, X. Wang, G. Diankov, H. Wang, and H. Dai, Nature Nanotech. 5, 321 (2010).

${ }^{13}$ X. Wang, Y. Ouyang, L. Jiao, H. Wang, L. Xie, J. Wu, J. Guo, and H. Dai, Nature Nanotech. 6, 563 (2011). 
${ }^{14}$ L. C. Campos, V. R. Manfrinato, J. D. Sanchez-Yamagishi, J. Kong, and P. Jarillo-Herrero, Nano Lett. 9, 2600 (2009).

${ }^{15}$ Z.-S. Wu, W. Ren, L. Gao, B. Liu, J. Zhao, and H.-M. Cheng, Nano Res. 3, 16 (2010).

${ }^{16}$ A. Chuvilin, E. Bichoutskaia, M. C. Gimenez-Lopez, T. Chamberlain, G. A. Rance, N. Kuganathan, J. Biskupek, U. Kaiser, and A. N. Khlobystov, Nature Mater. 10, 687 (2011).

${ }^{17}$ X. Jia, J. Campos-Delgado, M. Terrones, V. Meunier, and M. S. Dresselhaus, Nanoscale 3, 86 (2011).

${ }^{18}$ C. W. J. Beenakker, Rev. Mod. Phys. 69, 731 (1997).

${ }^{19}$ M. Berry and R. Mondragon, Proc. R. Soc. London A 412, 53 (1987).

${ }^{20}$ E. McCann, K. Kechedzhi, V. I. Fal'ko, H. Suzuura, T. Ando, and B. L. Altshuler, Phys. Rev. Lett. 97, 146805 (2006).

${ }^{21}$ A. F. Morpurgo and F. Guinea, Phys. Rev. Lett. 97, 196804 (2006).

${ }^{22}$ J. Wurm, A. Rycerz, I. Adagideli, M. Wimmer, K. Richter, and H. U. Baranger, Phys. Rev. Lett. 102, 056806 (2009).

${ }^{23}$ K. Wakabayashi, Y. Takane, and M. Sigrist, Phys. Rev. Lett. 99, 036601 (2007).

${ }^{24}$ K. Wakabayashi, Y. Takane, M. Yamamoto, and M. Sigrist, New J. Phys. 11, 095016 (2009).

${ }^{25}$ M. Yamamoto, Y. Takane, and K. Wakabayashi, Phys. Rev. B 79, 125421 (2009).

${ }^{26}$ H. Suzuura and T. Ando, Phys. Rev. Lett. 89, 266603 (2002).

${ }^{27}$ E. Mucciolo and C. Lewenkopf, J. Phys.: Condens. Matter 22, 273201 (2010).

${ }^{28}$ S. V. Morozov, K. S. Novoselov, M. I. Katsnelson, F. Schedin, L. A. Ponomarenko, D. Jiang, and A. K. Geim, Phys. Rev. Lett. 97, 016801 (2006).

${ }^{29}$ F. V. Tikhonenko, D. W. Horsell, R. V. Gorbachev, and A. K. Savchenko, Phys. Rev. Lett. 100, 056802 (2008).

${ }^{30}$ F. Ortmann, A. Cresti, G. Montambaux, and S. Roche, Europhys. Lett. 94, 47006 (2011).

${ }^{31}$ A. R. Akhmerov and C. W. J. Beenakker, Phys. Rev. Lett. 98, 157003 (2007).
${ }^{32}$ M. Wimmer and K. Richter, J. Comput. Phys. 228, 8548 (2009).

${ }^{33}$ We use a formulation similar to S. Sanvito, C. J. Lambert, J. H. Jefferson, and A. M. Bratkovsky, Phys. Rev. B 59, 11936 (1999).

${ }^{34}$ R. Landauer, IBM J. Res. Dev. 1, 223 (1957).

${ }^{35}$ M. Büttiker, Y. Imry, R. Landauer, and S. Pinhas, Phys. Rev. B 31, 6207 (1985).

${ }^{36}$ A. Rycerz, J. Tworzydło, and C. W. J. Beenakker, Europhys. Lett. 79, 57003 (2007).

${ }^{37}$ C. H. Lewenkopf, E. R. Mucciolo, and A. H. Castro Neto, Phys. Rev. B 77, 081410 (2008).

${ }^{38}$ V. Krueckl and T. Kramer, New J. Phys. 11, 093010 (2009).

${ }^{39}$ J. Wurm, M. Wimmer, H. U. Baranger, and K. Richter, Semicond. Sci. Technol. 25, 034003 (2010).

${ }^{40}$ F. Libisch, S. Rotter, and J. Burgdörfer, Phys. Status Solidi B 248 , 2598 (2011); arXiv:1104.5260v1.

${ }^{41}$ A. R. Akhmerov, J. H. Bardarson, A. Rycerz, and C. W. J. Beenakker, Phys. Rev. B 77, 205416 (2008).

${ }^{42}$ P. M. Ostrovsky, I. V. Gornyi, and A. D. Mirlin, Eur. Phys. J. Spec. Top. 148, 63 (2007).

${ }^{43}$ The most general form is in fact $\mathcal{T}_{0 z}(\vartheta, \phi)=\left(\cos \vartheta e^{i \phi} \tau_{0}+\right.$ $\left.\sin \vartheta e^{i(\pi / 2+\phi)} \tau_{z}\right) \otimes \sigma_{y} \mathcal{C}$. The phase freedom $\phi$ does not play a role in our analysis and we set $\phi=0$ in the main text.

${ }^{44}$ F. Evers and A. D. Mirlin, Rev. Mod. Phys. 80, 1355 (2008).

${ }^{45}$ E. McCann and V. I. Fal'ko, J. Phys.: Condens. Matter 16, 2371 (2004).

${ }^{46}$ J. Wurm, K. Richter, and I. Adagideli, Phys. Rev. B 84, 075468 (2011).

${ }^{47}$ T. Luo, A. P. Iyengar, H. A. Fertig, and L. Brey, Phys. Rev. B 80, 165310 (2009)

${ }^{48}$ Note that $\mathcal{T}_{y}, \mathcal{T}_{0 z}(\vartheta)$ and the pseudovalley structure $\boldsymbol{v} \cdot \boldsymbol{\tau}$ are not independent, as $\mathcal{T}_{0 z}(\vartheta) \mathcal{T}_{y}=\boldsymbol{v} \cdot \boldsymbol{\tau}$. Hence, $\mathcal{T}_{y}$ and $\mathcal{T}_{0 z}(\vartheta)$ carry the same information.

${ }^{49}$ R. Jackiw and C. Rebbi, Phys. Rev. D 13, 3398 (1976).

${ }^{50}$ G. W. Semenoff, V. Semenoff, and F. Zhou, Phys. Rev. Lett. 101, 087204 (2008).

${ }^{51}$ T. Ando, J. Phys. Soc. Jpn. 74, 777 (2005). 